PREPARED FOR THE U.S. DEPARTMENT OF ENERGY, UNDER CONTRACT DE-AC02-76CH03073

PPPL-3904

PPPL-3904

UC-70

Destruction of Invariant Surfaces and Magnetic Coordinates for Perturbed Magnetic Fields

by

S.R. Hudson

November 2003

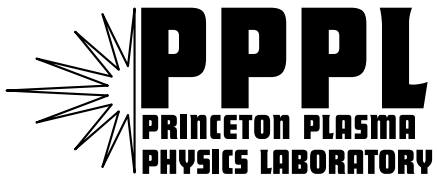

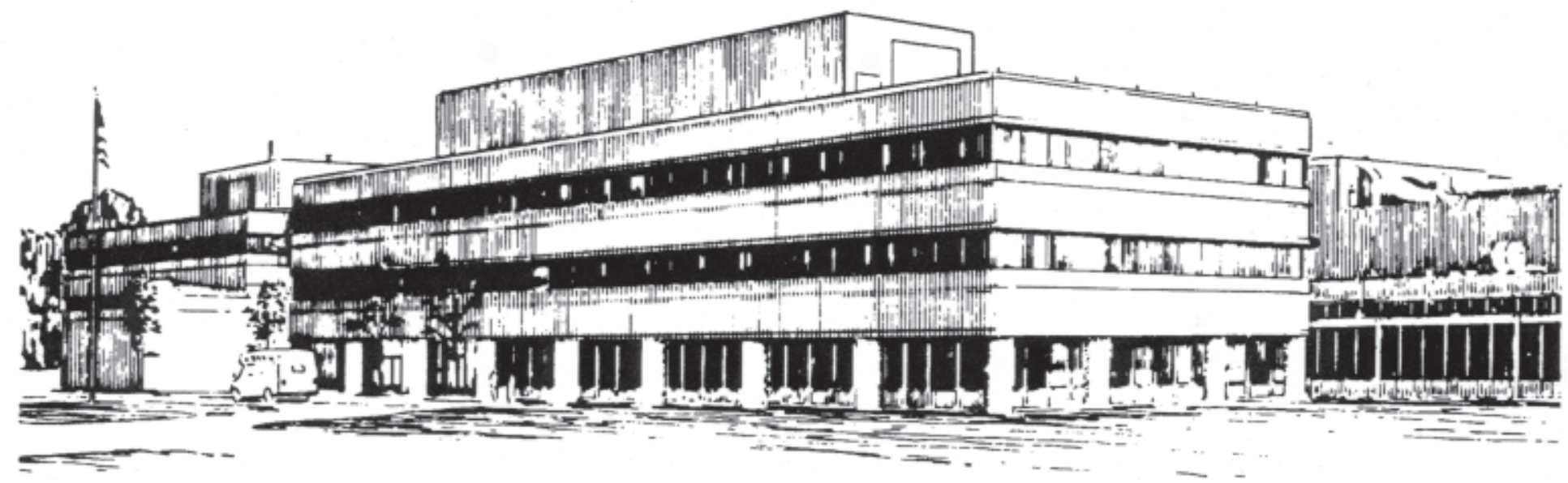

PRINCETON PLASMA PHYSICS LABORATORY PRINCETON UNIVERSITY, PRINCETON, NEW JERSEY 


\section{PPPL Reports Disclaimer}

This report was prepared as an account of work sponsored by an agency of the United States Government. Neither the United States Government nor any agency thereof, nor any of their employees, makes any warranty, express or implied, or assumes any legal liability or responsibility for the accuracy, completeness, or usefulness of any information, apparatus, product, or process disclosed, or represents that its use would not infringe privately owned rights. Reference herein to any specific commercial product, process, or service by trade name, trademark, manufacturer, or otherwise, does not necessarily constitute or imply its endorsement, recommendation, or favoring by the United States Government or any agency thereof. The views and opinions of authors expressed herein do not necessarily state or reflect those of the United States Government or any agency thereof.

\section{Availability}

This report is posted on the U.S. Department of Energy's Princeton Plasma Physics Laboratory Publications and Reports web site in Fiscal Year 2004. The home page for PPPL Reports and Publications is: http://www.pppl.gov/pub_report/

DOE and DOE Contractors can obtain copies of this report from:

U.S. Department of Energy

Office of Scientific and Technical Information

DOE Technical Information Services (DTIS)

P.O. Box 62

Oak Ridge, TN 37831

Telephone: (865) 576-8401

Fax: (865) 576-5728

Email: reports@adonis.osti.gov

This report is available to the general public from:

National Technical Information Service

U.S. Department of Commerce

5285 Port Royal Road

Springfield, VA 22161

Telephone: $1-800-553-6847$ or

(703) $605-6000$

Fax: (703) 321-8547

Internet: http://www.ntis.gov/ordering.htm 


\title{
Destruction of invariant surfaces and magnetic coordinates for perturbed magnetic fields
}

\author{
S.R.Hudson \\ Princeton Plasma Physics Laboratory, PO Box 451, Princeton NJ 08543, USA.
}

November 17, 2003

\begin{abstract}
Straight-field-line coordinates are constructed for nearly integrable magnetic fields. The coordinates are based on the robust, noble-irrational rotational-transform surfaces, whose existence is determined by an application of Greene's residue criterion. A simple method to locate these surfaces is described. Sequences of surfaces with rotational-transform converging to low order rationals maximize the region of straight-field-line coordinates.
\end{abstract}




\section{Introduction}

As toroidal magnetic field line flow, with non-vanishing toroidal component, is a Hamiltonian system $[1,2]$, all the powerful and insightful tools valid for Hamiltonian systems may be applied (see Refs. $[3,4]$ for comprehensive discussions of dynamical systems). A particularly useful property is that, if the system is integrable, then action-angle coordinates may be constructed everywhere [5]. The analog of action-angle coordinates for magnetic field line flow is straight-field-line coordinates. Such coordinates greatly simplify the dynamics. Along a field line in an integrable system, the 'action' coordinate, $\psi$ (toroidal flux), is constant and the 'angle' coordinate, $\alpha$ (poloidal angle), increases linearly with the 'time' coordinate, $\zeta$ (toroidal angle), as $\alpha(\zeta)=\alpha_{0}+t(\psi) \zeta$, where $t$ is the rotational-transform. As plasma dynamics is strongly influenced by the confining magnetic field, it is not surprising that straight-field-line coordinates are widely used in plasma physics, with the most common choice being Boozer coordinates [6].

Straight-field-line coordinates can only be constructed on flux-surfaces. For integrable fields, all the magnetic field lines lie on flux-surfaces. For a non-integrable field the situation is more complicated. A non-integrable field may be considered as an integrable field plus a non-integrable perturbation and is the realistic case for plasma confinement devices. Magnetic islands form at the rational rotational-transform surfaces and chaotic trajectories emerge near unstable periodic orbits. In such regions, straight-field-line coordinates cannot be constructed. Nonetheless, the usefulness of straight-field-line coordinates is not completely lost. Depending on the magnitude of the perturbation, the Kolmogorov-Arnold-Moser (KAM) theorem $[7,8,9]$ shows that fluxsurfaces with sufficiently irrational rotational-transform survive perturbation. These flux surfaces

are commonly called KAM surfaces. If the integrability destroying perturbation is small, then 
flux surfaces will exist almost everywhere.

The construction of straight-field-line coordinates becomes increasingly treacherous as the perturbation strength increases. The existence of a given flux-surface is fragile. The irrational rotational-transform surfaces will disintegrate into invariant Cantor sets (cantori) for strong perturbation and the region occupied by chaotic field lines will increase. Considering that both the rational and irrationals are dense in the set of real numbers $[10,11]$, the structure of the magnetic field, at every level of detail, is a complex mixture of periodic orbits, island chains and chaotic trajectories interspersed with invariant surfaces and cantori.

In the plasma physics community there are several computational codes that consider the challenging problem of describing plasma behavior in perturbed and chaotic magnetic fields $[12,13,14,15]$. While it is not necessary to use straight-field-line coordinates, it is helpful to be aware of the type of behavior that chaotic magnetic fields exhibit.

This article will present a construction of straight-field-line coordinates for nearly integrable fields containing magnetic islands, chaotic field lines and invariant flux surfaces. The coordinates are based on a selection of flux surfaces with noble irrational rotational-transform. These surfaces are likely to be most robust to chaos. A sensitive technique is employed (Greene's residue criterion) to determine if a chosen surface persists or has been destroyed by perturbation. The construction of each surface employs a method that explicitly locates a surface with desired rotational-transform, without numerically calculating the rotational-transform by field line following.

The rotational-transform $t$ is defined as the limit

$t=\lim _{\Delta \zeta \rightarrow \infty} \frac{\Delta \theta}{\Delta \zeta}$ 
where $\theta$ is an arbitrary poloidal angle coordinate. This limit exists if, for any given positive $\delta$, there exists a $\Delta \zeta_{\delta}$ such that for all $\Delta \zeta>\Delta \zeta_{\delta},|\Delta \theta / \Delta \zeta-t|<\delta$. For a chaotic trajectory, it is not clear that this limit exists. A given chaotic field line may spend an arbitrarily long time in a certain region of space with a certain average transform, then squeeze through a partial barrier (cantori) into a different region with a different average transform. An appeal to the ergodic theorem, where the 'time' average is equal to the accessible phase space average, may provide some relief, but for practical purposes in which a field line cannot be followed an 'infinite' distance (about $10^{10}$ transits in [16]), and considering the complex structure of chaotic fields, any practical measurement of the rotational-transform will be unreliable in chaotic regions. Furthermore, in chaotic regions, nearby trajectories will diverge exponentially at a rate characterized by the Lyapunov exponent [3]. It is likely that any finite approximation to the rotational-transform limit will be a non-monotonic function of position in the chaotic region. In regular regions of space occupied predominately with flux surfaces, the situation is different. On KAM surfaces, the limit will converge to arbitrary accuracy if the field line is followed a sufficient distance and efficient methods for calculating the transform exist $[17,18,19]$. Finally, for periodic field lines, which close after a finite number of transits, the rotational-transform can be determined exactly after following the field line a finite distance.

The outline of this article is as follows. Sec.(II) will describe the theoretical and numerical tools that will be applied. In particular, the representation of magnetic field line flow as a mapping, the tangent mapping, stellarator symmetry, and the continued fraction representation will be briefly described. Also, Greene's criterion for determining the existence of a given irrational surface is described. Sec.(III) will present a selection of an 'optimal' set of surfaces by choosing the rotational-transform. In Sec.(IV), the existence of each selected surface is determined using 
Greene's criterion, and in Sec.(V) an explicit construction of an invariant surface is presented. Having i)selected, ii)determined the existence of, and iii)constructed a suitable set of invariant surfaces, magnetic coordinates are then obtained in Sec.(VI). By selecting the most-irrational surfaces, the magnetic coordinates thus constructed are likely to be those most robust to chaos. Finally, some comments are given in Sec.(VII).

\section{Preliminaries}

Straight-field-line coordinates are determined by the magnetic field. This article will consider the magnetic field to be given numerically in the form

$\mathbf{B}=B^{\rho}(\rho, \theta, \phi) \mathbf{e}_{\rho}+B^{\theta}(\rho, \theta, \phi) \mathbf{e}_{\theta}+B^{\phi}(\rho, \theta, \phi) \mathbf{e}_{\phi}$

where $(\rho, \theta, \phi)$ form a toroidal coordinate system, $\mathbf{e}_{\rho}=\partial_{\rho} \mathbf{x}, \mathbf{e}_{\theta}=\partial_{\theta} \mathbf{x}$, and $\mathbf{e}_{\phi}=\partial_{\phi} \mathbf{x}$ where $\mathbf{x}$ is the position vector and $B^{\phi}$ is nowhere zero in the region of interest. This article will consider fields that are consistent with stellarator symmetry [20]. The magnetic field and coordinates are stellarator symmetric if

$$
\begin{aligned}
B^{\rho}(\rho, \theta, \phi) & =-B^{\rho}(\rho,-\theta,-\phi), \\
B^{\theta}(\rho, \theta, \phi) & =B^{\theta}(\rho,-\theta,-\phi), \\
B^{\zeta}(\rho, \theta, \phi) & =B^{\zeta}(\rho,-\theta,-\phi) .
\end{aligned}
$$

It follows that if $\{\rho(\phi), \theta(\phi)\}$ is a field line then so also is $\{\rho(-\phi),-\theta(-\phi)\}$. Stellarator symmetry is equivalent to time-reversal symmetry in dynamical systems.

A field line is determined by numerically integrating

$$
\frac{d \rho}{d \phi}=\dot{\rho}(\rho, \theta, \phi)=\frac{B^{\rho}}{B^{\phi}}
$$


$\frac{d \theta}{d \phi}=\dot{\theta}(\rho, \theta, \phi)=\frac{B^{\theta}}{B^{\phi}}$

from a given starting point. The magnetic field line flow produces a mapping

$$
\left(\begin{array}{c}
\tilde{\rho} \\
\tilde{\theta}
\end{array}\right)=T\left(\begin{array}{l}
\rho \\
\theta
\end{array}\right),
$$

where the mapping $T$ integrates the field lines over one toroidal period from a given starting point, typically chosen on a symmetry plane.

Periodic orbits serve as a useful framework for understanding chaotic fields and are defined as orbits that satisfy

$\rho(\phi+2 \pi q / N)=\rho(\phi)$

$\theta(\phi+2 \pi q / N)=\theta(\phi)+2 \pi p$

for integers $(p, q)$ and where $N$ is the toroidal periodicity of the device.

The search for symmetric periodic orbits is greatly simplified for stellarator symmetric fields. By writing the mapping $T$ as a product of involutions (which is possible for stellarator symmetric fields; see $[21,3,22]$ for details), it is only required to search for orbits that satisfy

$\theta(\phi+\pi q / N)=\theta(\phi)+\pi p$

on the $\theta=0$ line. Such symmetric periodic orbits are located via a one-dimensional search (note that derivative information is available from the tangent map described below). This halves the computational effort and affords greater numerical accuracy. 
The behavior of orbits near a given orbit is described by the tangent map [3]

$$
\left(\begin{array}{c}
\delta \tilde{\rho} \\
\delta \tilde{\theta}
\end{array}\right)=M\left(\begin{array}{c}
\delta \rho \\
\delta \theta
\end{array}\right)
$$

where $M$ is defined

$$
M=\left(\begin{array}{cc}
\partial_{\rho} \tilde{\rho}, & \partial_{\theta} \tilde{\rho} \\
\partial_{\rho} \tilde{\theta}, & \partial_{\theta} \tilde{\theta}
\end{array}\right)
$$

and is determined by field line integration

$$
\frac{d M}{d \phi}=\left(\begin{array}{cc}
\partial_{\rho} \dot{\rho}, & \partial_{\theta} \dot{\rho} \\
\partial_{\rho} \dot{\theta}, & \partial_{\theta} \dot{\theta}
\end{array}\right) M
$$

The full-period tangent mapping $M^{q}$ at a periodic orbit determines the stability of the orbit [3]. If the eigenvalues of $M^{q}$ are complex conjugates, the tangent orbits will display elliptical motion under the mapping near the periodic orbit and that periodic orbit is considered stable. If the eigenvalues are real reciprocals, the tangent motion will either exponentially grow or decay and the periodic orbit is unstable.

Greene [21] suggested that the existence of a given irrational KAM surface is related to the stability of neighboring periodic orbits — in particular the periodic orbits that 'best approximate' the irrational. The term 'best approximate' is made clear using the continued fraction representation of the real numbers. Any real number $t$ may be expressed as

$$
t=a_{0}+\frac{1}{a_{1}+\frac{1}{a_{2}+\frac{1}{a_{3}+\ldots}}}=\left[a_{0}, a_{1}, a_{2}, a_{3}, \ldots\right]
$$

where the integers $a_{m}$ are called the partial quotients. The continued fraction representation is described in detail by Niven [11]. Some salient points are reproduced here for completeness: every 
irrational has a unique representation as an infinite continued fraction; the sequence will terminate if $t$ is rational and will continue infinitely if $t$ is irrational; the rational $p_{m} / q_{m}=\left[a_{0}, a_{1}, a_{2}, \ldots, a_{m}\right]$ is called the $m$ th convergent; the convergents form a sequence of consecutively better approximates that are the 'best' in the sense that if $|t-a / b|<\left|t-p_{m} / q_{m}\right|$ where $a, b$ are integers, then $b>q_{m}$; and successive convergents bound the irrational $p_{m} / q_{m}<t<p_{m+1} / q_{m+1}$ (or $p_{m+1} / q_{m+1}<t<$ $p_{m} / q_{m}$ depending on $m$ ). A real number is determined by the continued fraction representation by $t=\alpha_{0}$ where $\alpha_{0}$ is defined inductively by the partial quotients

$\alpha_{n}=a_{n}+\frac{1}{\alpha_{n+1}}$

The magnitude of the partial quotients $a_{m}$ indicates how rapidly the $p_{m} / q_{m}$ converge to $t$. Irrationals with continued fraction representations terminating in infinitely many 1 's, $\left[a_{0}, \ldots, a_{m}, 1,1\right.$, $1, \ldots]$, are most difficult to approximate with rationals, in the sense that higher order rationals are required to achieve a given accuracy, and give meaning to the expression 'most-irrational'. Such irrationals are commonly called noble irrationals. The most noble irrational is $[1,1,1,1,1, \ldots]$, and by setting $\alpha_{n}=\alpha_{n+1}$ in Eqn.(14), this value is determined $\gamma=(1+\sqrt{5}) / 2$. Interestingly, this number, called the golden mean, is the limiting ratio of the Fibonacci series. There is a close relationship between the continued fraction representation and the Farey tree [23]. The continued fraction representation for a rational is unique with the following exception

$p / q=\left[a_{0}, a_{1}, \ldots, a_{m}\right]=\left[a_{0}, a_{1}, \ldots, a_{m}-1,1\right]$.

The idea of Greene's method is that a given irrational surface will exist if the sequence of convergents are stable. Greene presented this method as a conjecture. It has nonetheless yielded impressive results and MacKay has discussed the reliability of the assumptions [24]. Greene con- 
sidered a quantity called the residue, $R$, which characterizes the stability of a periodic orbit. It is defined by the tangent map evaluated along the full periodic orbit by

$R=\frac{2-\operatorname{Tr}\left(M^{q}\right)}{4}$

For the purposes of this article is it sufficient to note the following. For a given periodic orbit, the residue is a function of the perturbation. If the residues of the convergents of a given irrational approach zero, then that irrational surface will exist. If, however, the residues become large, then that irrational surface has been destroyed. The critical value is $R=0.25$ : if the residues of the convergents approach 0.25 then the irrational surface is on the edge of destruction. By criticality it is meant that the KAM surface is on the verge of destruction. The behavior of $R$ at criticality is interesting topic: for example, for the standard non-twist map [26], the residue converges to a 6-cycle [22]. Such matters are beyond the scope of the present application and it is sufficient to determine if the residues are large or small compared to 0.25 .

Considering the residue to be a function of the continued fraction representation, at fixed perturbation, Greene noted that

$R\left(\left[a_{0}, \ldots, a_{i}+1, \ldots, a_{N}\right]\right)>R\left(\left[a_{0}, \ldots, a_{i}, \ldots, a_{N}\right]\right)$

where $a_{i}=\max _{j}\left(a_{j}\right)$. Loosely speaking, rationals with smaller partial quotients (described in this article are more-noble) have smaller residue: the more-noble irrationals are more robust to chaos. Also, Greene noted that $R \rightarrow \infty$ as $a_{i} \rightarrow \infty$, implying that there is a band of chaos associated with every rational.

That the noble irrationals are most robust to perturbation may be understood by noting that they are farthest from low-order rationals and thus least likely to be destroyed by island overlap 
— a mechanism for chaos suggested by Chirikov [25].

Greene's residue criterion gives a very sensitive and clear criterion for determining the existence of an irrational surface. Furthermore, it guides selection of the most suitable surfaces to be used as a coordinate framework in Sec.(III). The residue has been applied in the context of magnetic field line flow by Hanson and Cary [27, 28].

Not only does the residue criterion enable the existence of a given irrational surface to be determined, it can also be used to predict whether any invariant irrational surface exists in a given interval. In this context the residue criterion may be used to identify and locate the invariant surface which defines the boundary of a chaotic region [29]. Such a surface may be called the boundary surface. Consider the interval $\left[p_{0} / q_{0}, p_{1} / q_{1}\right]$, where the rationals $p_{0} / q_{0}, p_{1} / q_{1}$ are neighboring, $p_{1} q_{0}-p_{0} q_{1}= \pm 1$, and the mediant $p / q=\left(p_{0}+p_{1}\right) /\left(q_{0}+q_{1}\right)$. By a criterion of Ref.[29], invariant surfaces with rotational-transform in $\left[p_{0} / q_{0}, p_{1} / q_{1}\right]$ ( are / are not) likely to exist if the average residue $\left(R_{\left(p_{0}, q_{0}\right)}+R_{\left(p_{1}, q_{1}\right)}\right) / 2$ is significantly ( smaller / larger ) than 0.25 . By recursively subdividing the interval using the mediant, and testing the ( lower / upper ) subinterval for the existence of invariant surfaces, an algorithm is devised that will ultimately lead to the invariant surface with the ( lowest / highest ) rotational-transform in the original interval. At each subdivision, the interval length is reduced. Furthermore, as the rationals $p_{0} / q_{0}, p_{1} / q_{1}$ are neighboring, the successive rationals have a natural connection to the convergents of nearby irrationals. This allows the continued fraction representation of the boundary surface to be deduced [29]. Alternatively, the algorithm is terminated at some point (perhaps when the periodic orbits become too long and numerical accuracy becomes poor) and the locally most robust irrational surface [30] is given by $\left(p_{n}+\gamma p_{n+1}\right) /\left(q_{n}+\gamma q_{n+1}\right)$. This method for constructing the boundary surface will be used in Sec.(VI). 
The model magnetic field used for this study is given as

$\mathbf{B}=\nabla \rho \times \nabla \theta+\nabla \phi \times \nabla \chi(\rho, \theta, \phi)$

where the field line Hamiltonian $\chi$ is given

$\chi=\rho^{2} / 2+k\left[\frac{1}{2} \cos (2 \theta-\phi)+\frac{1}{3} \cos (3 \theta-2 \phi)\right]$.

The equations governing the field line are then

$\dot{\rho}=-k[\sin (2 \theta-\phi)+\sin (3 \theta-2 \phi)]$,

$\dot{\theta}=\rho$.

This field is integrable, and $(\rho, \theta, \phi)$ are action-angle coordinates, if the perturbation parameter $k$ is zero. In this case, the rotational-transform profile is $t=\rho$. For non-zero $k$, 'primary' islands will form at the $p_{1} / q_{1}=1 / 2, p_{2} / q_{2}=2 / 3$ surfaces. As $k$ is increased, 'secondary' islands will form at all rationals on the Farey tree formed by $p_{1} / q_{1}$ and $p_{2} / q_{2}$ (see [23] for a description of the Farey tree), chaotic regions will develop and invariant surfaces will be destroyed.

A generally perturbed field will have a perturbation spectrum that is initially unknown. The model field presented here has sufficient complexity to represent a generally perturbed chaotic field relevant for fusion confinement devices, with the exception of reversed shear systems for which a model non-twist map [26] is required.

The remainder of this article will use these ideas i)to select, ii)to determine the existence of, and iii)to construct an appropriate set of irrational surfaces. On these surfaces, magnetic coordinates will be constructed. 


\section{Surface selection}

The selection of surfaces is somewhat arbitrary and may be adapted to suit the particular application. In the construction of magnetic coordinates to be described in Sec.(VI), it is assumed that the chaotic field lines are associated with a few low-order islands. We thus seek a selection of surfaces that will maximize the region of straight-field-line coordinates — that is, lie as close as possible to the chaotic regions of the low-order islands - and that the selection is based on the most robust irrational surfaces.

The non-uniqueness of the continued fraction representation of a rational is convenient for constructing sequences of noble irrationals that converge from above and below any given rational $p / q=\left[a_{0}, a_{1}, \ldots, a_{m}\right]$. The sequences of noble irrationals

$$
\begin{aligned}
t & =\left[a_{0}, a_{1}, \ldots, a_{m}, n, 1,1,1, \ldots\right] \\
t & =\left[a_{0}, a_{1}, \ldots, a_{m}-1,1, n, 1,1, \ldots\right]
\end{aligned}
$$

will converge from above or below, depending on whether $m$ is odd or even, to $p / q$ as $n$ increases. The 'nobility' of the irrationals in these sequences decreases as $n$ increases. As $n$ increases, the location of the irrational surfaces will approach the chaotic region near the $p / q$ unstable orbit and at some point will be engulfed by the associated chaos.

These two sequences alone may not provide sufficient spatial resolution, but arbitrarily many such sequences, defined by a fixed set of integers $\left\{n_{1}, n_{2}, \ldots, n_{N}\right\}$ may be constructed

$$
\begin{aligned}
t & =\left[a_{0}, \ldots, a_{m}, n, n_{1}, n_{2}, \ldots, n_{N}, 1,1, \ldots\right] \\
t & =\left[a_{0}, \ldots, a_{m}-1,1, n, n_{1}, n_{2}, \ldots, n_{N}, 1,1, \ldots\right] .
\end{aligned}
$$

This is equivalent to appending an infinite sequence of 1's to the continued fraction representation of a rational. For fixed $\left\{n_{1}, n_{2}, \ldots, n_{N}\right\}$, each such sequence will converge to the rational $p / q=\left[a_{0}\right.$, 
$\left.a_{1}, \ldots, a_{m}\right]$ as $n$ increases. Using such sequences, an arbitrarily dense set of noble irrationals that converge to a given rational can be constructed.

Without a-priori knowledge of the perturbation spectrum, it cannot be pre-determined exactly which irrational surfaces will survive perturbation. It cannot be guaranteed that a given selection of irrational surfaces is the most robust to perturbation, but the sequences as defined can be expected to be the most robust as they are based on the most noble irrational KAM surfaces. This selection adaptively expands to fill the region in which invariant tori exist and serves to partition regions of chaos.

\section{Surface existence}

It is not necessary to pre-determine the existence of a selected surface. One may proceed to directly construct the surface and then determine a-posteriori if that surface was successfully constructed. However, the efficiency and reliability of Greene's method for determining the existence of an irrational flux surface justifies the additional computational cost. In fact, as any numerical method for constructing an invariant surface will fail if that invariant surface does not exist, pre-determining the existence of a required surface may save computational effort.

To illustrate the application of Greene's residue criterion, the critical perturbation is determined for the surface with rotational-transform equal to the inverse golden mean $\gamma^{-1}=[0,1,1,1, \ldots]$. The convergents of $\gamma^{-1}$ are $1 / 2,2 / 3,3 / 5,5 / 8,8 / 13,13 / 21,21 / 34,34 / 55,55 / 89,89 / 144,144 / 233$, $233 / 377,377 / 610, \ldots$. The behavior of the residue of the convergents for three perturbation values — below, near and above criticality — is shown in Fig.(1). 
For less-than-critical perturbation, the residues approach zero. For near-critical perturbation, the residues approach 0.25 ; and for larger-than-critical perturbation, the residues become large. For these three cases, detailed Poincaré plots Fig.(2),Fig.(3),Fig.(4) confirm that the residue criterion predicts the breakup of the surface. In each of the Poincaré plots, the location of the $(89,144)$ periodic orbit is shown with $\triangle$, the $(144,233)$ with $\square$, the $(233,377)$ with $\times$, and the $(377,610)$ is shown with + . If the invariant surface exists, it will be located between successive convergents and thus lie between the $\times$ 's and the +'s. In Fig.(4), above criticality, this region has become chaotic.

To illustrate that the more-noble irrationals are more robust to chaos, the critical perturbation for each of the irrationals comprising the sequences given in Table (I) is determined. For all these sequences, as $n$ increases the irrationals converge, either from above or below, to the limit rational. As $n$ increases the 'nobility' of the irrational decreases and it is expected that the larger $n$ irrationals will be destroyed at lower perturbation. These expectations are consistent with the numerical determination of the critical perturbation parameter $k$ for each irrational, as shown in Fig.(5). This fractal 'critical-function' has been studied by several authors for the standard map (see for example Ref.[31]). For each irrational, the residue of the first 13 convergents is calculated in the application of Greene's criterion.

To further illustrate the behavior, the critical perturbation parameter for the following irrationals is determined

$$
\begin{aligned}
t & =[0,1,1, n, j, 1,1,1,1, \ldots], \\
t & =[0,1,1,1, n, j, 1,1,1, \ldots],
\end{aligned}
$$

where $j$ is an arbitrary, fixed positive integer. These irrationals converge to $1 / 2$ and $2 / 3$ as $n$ 
increases. Sequences with $j>1$ may be considered less-noble than the sequence with $j=1$, and indeed the less-noble irrationals are destroyed at lower perturbation as shown in Fig.(6). For this figure, the critical perturbation parameters for irrational sequences of the type given in Eqn.(24) with $j=1,5,10$ are compared. Note that the less-noble irrationals, that is those with $j=5,10$, are interspersed among the most-noble irrationals; thus, at certain perturbation parameters, between two irrational surfaces that are invariant, there are irrational surfaces that have been destroyed. This highlights the perils of interpolating straight-field-line coordinates between invariant surfaces.

\section{Surface construction}

Given that the preferred surfaces have been identified, and that their existence may be determined, a method to construct a surface of prescribed irrational rotational-transform will now be described. This construction is similar to the method proposed by Dewar and Meiss [32], in that a curve of prescribed transform is defined by minimizing the difference between the curve and its own image under the map. In this case, a Newton method is used to find the curve that exactly coincides with its image, which is thus an invariant curve. Newton methods for finding invariant surfaces have also been used by Reiman and Pomphrey [33], but they did not constrain the rotational-transform of the surface.

A continuous arbitrary trial curve is parameterized with a poloidal angle $\alpha$ using a Fourier representation

$$
\begin{aligned}
\rho(\alpha) & =\sum_{m=0}^{M} \rho_{m} \cos (m \alpha), \\
\theta(\alpha) & =\alpha+\sum_{m=1}^{M} \theta_{m} \sin (m \alpha) .
\end{aligned}
$$


The parameter $\alpha$ will be identified with the straight-field-line poloidal coordinate. This curve may be considered as the intersection of an invariant surface, with 'action' coordinate $\psi$ and specified rotational-transform $t(\psi)$, with the plane $\phi=0$. The even representation (cosine series) of the radial coordinate $\rho$ and the odd representation (sine series) of the poloidal coordinate $\theta$ (in addition to the secular term $\alpha$ ) is consistent with the assumption of a stellarator symmetric field and coordinates. This curve, and therefore the surface defined by allowing the curve to flow with the magnetic field, is invariant if it is mapped to itself under the field line flow.

A discrete set of points $\left(\rho_{i}, \theta_{i}\right)$ is constructed equally spaced in $\alpha$

$\left\{\alpha \mid \alpha_{i}=2 \pi i / N, i=0, N-1\right\}$

and is mapped to $\left(\tilde{\rho}_{i}, \tilde{\theta}_{i}\right)$ by $T$.

By requiring the trial curve to be invariant under the rigid rotation map $(\psi, \alpha) \mapsto(\psi, \alpha+2 \pi t)$, the discrete set of points $\left(\rho_{i}, \theta_{i}\right)$ maps to

$\bar{\rho}_{i}=\sum_{m=0}^{M} \rho_{m} \cos \left[m\left(\alpha_{i}+2 \pi t\right)\right]$

$\bar{\theta}_{i}=(\alpha+2 \pi t)+\sum_{m=1}^{M} \theta_{m} \sin \left[m\left(\alpha_{i}+2 \pi t\right)\right]$.

Constructing the 'error' vector $\mathbf{f}=\left\{\bar{\rho}_{i}-\tilde{\rho}_{i}, \bar{\theta}_{i}-\tilde{\theta}_{i}\right\}$, which has length $2 N$, to be a function of $\mathbf{x}=\left\{\rho_{m}, \theta_{m}\right\}$, which has length $2 M+1$, a Newton correction for the invariant curve is found by solving for $\delta \mathbf{x}$

$0=\mathbf{f}(\mathbf{x})+\mathbf{d f} \cdot \delta \mathbf{x}$

The derivative matrix $\mathbf{d f}$ is provided by the tangent map

$\partial_{\rho_{m}}\left(\bar{\rho}_{i}-\tilde{\rho}_{i}\right)=\partial_{\rho_{m}} \bar{\rho}_{i}-\partial_{\rho} \tilde{\rho}_{i} \partial_{\rho_{m}} \rho$ 
$\partial_{\theta_{m}}\left(\bar{\rho}_{i}-\tilde{\rho}_{i}\right)=-\partial_{\theta} \tilde{\rho}_{i} \partial_{\theta_{m}} \theta$,

$\partial_{\rho_{m}}\left(\bar{\theta}_{i}-\tilde{\theta}_{i}\right)=-\partial_{\rho} \tilde{\theta}_{i} \partial_{\rho_{m}} \rho$

$\partial_{\theta_{m}}\left(\bar{\theta}_{i}-\tilde{\theta}_{i}\right)=\partial_{\theta_{m}} \bar{\theta}_{i}-\partial_{\theta} \tilde{\theta}_{i} \partial_{\theta_{m}} \theta$

and is inverted using singular value decomposition (SVD) [34].

By choosing $2 N$ to be greater than $2 M+1$, the SVD method will minimize $|f|$, whether the surface indeed exists or not. The iterations are terminated when $|\delta \mathbf{x}|$ is smaller than some prescribed tolerance. With Newton methods, it is required to provide an initial guess for the iterations. This may be provided by knowledge of the rotational-transform profile. Alternatively, the location of the convergents will approximate the irrational curve.

A Fourier decomposition of the entire surface in straight-field-line coordinates is immediately possible by allowing the invariant curve to flow along the field one period. This point will be expanded in the following section.

The accuracy to which the invariant surface is constructed is dependent on the resolution of the Fourier representation. A convenient way to determine if sufficiently many harmonics have been used is to confirm that the Fourier representation of the invariant surface lies between the convergent periodic orbits. If not, then though the invariant surface may indeed exist, insufficient Fourier resolution has been used. Other methods to establish the surface has been successfully constructed include confirming that $|f|$ is close to zero, that the Fourier modes decay sufficiently rapidly compared to $M$, and that the measured rotational-transform of a field line on the surface agrees with the prescribed value. 


\section{Straight field line coordinates}

The necessary ingredients for a robust construction of magnetic coordinates are now prepared. In this section, it will be assumed that the chaotic trajectories arise near a few low-order periodic orbits. Sequences of noble-irrationals of the type given in Eqn.(22) will be used to select which surfaces will be used.

Straight-field-line coordinates can only be constructed on invariant surfaces. The coordinates may be interpolated, but between invariant surfaces there will, in general, be an island chain and associated band of chaos and the interpolated coordinates will not be straight-field-line coordinates. For low-order rationals, the width of the region contained by islands and chaos may be significant. For high-order rational surfaces, the chaotic region may become vanishingly small. In this case, the interpolated coordinates, to a sufficient approximation, may be deemed to be straight-field-line coordinates.

A selection of noble-irrational surfaces converging to $(2 / 3)$ from below as $n$ increases

$t=[0,1,1,1, n, 1,1,1,1, \ldots]$

is used to construct magnetic coordinates and these are shown in Fig.(7). For this figure, only the region near the $(2 / 3)$ unstable periodic orbit is shown. In the left side of this figure, the surfaces that are deemed to exist (that have convergents with residue approaching zero) are constructed and plotted with solid lines, and for such surfaces the straight-field-line angle grid is shown. As $n$ increases, the surfaces approach the chaotic region and for some $n$ will be destroyed. The last surface of this sequence that is deemed to exist has rotational transform $t=0.65682049400299220=[0,1,1,1,10,1,1,1, \ldots]$ 
The boundary surface is not required to be a member of this sequence. The algorithm for locating boundary surfaces is applied to the interval defined by the rationals $p_{0} / q_{0}=21 / 32=$ $[0,1,1,1,10]$ and $p_{1} / q_{1}=2 / 3=[0,1,1,1]$. After 10 subdivisions of this interval, the invariant surface that is closest to the $(2 / 3)$ chaotic region is determined to have rotational transform $t=0.65696574415284240=[0,1,1,1,10,1,3,1,2,3,1,1,1, \ldots]$. This is also shown with a solid line in the left side of the figure. To distinguish this surface, the angle coordinate grid is not extended to this surface.

On all the invariant surfaces, a field line is followed 5000 toroidal periods and is plotted on both sides of the figure. These orbits coincide with the invariant surfaces. Also shown are some orbits in the chaotic region to illustrate the stochastic region. In this application, $M=100$ Fourier modes were used to describe the surfaces and the perturbation parameter $k=0.5 \times 10^{-3}$. In determining the surfaces, the total number of $T$ evaluations is approximately $5 N$, where $N>(2 M+1)$, and thus the method is computationally competitive with field line tracing methods.

The global nature of the magnetic coordinates is displayed in Fig.(8). For this construction, sequences of noble irrationals approaching $(1 / 2)$ and $(2 / 3)$ as $n$ increases were used as the coordinate framework

$$
\begin{aligned}
t & =[0,2, n, 1,1,1,1, \ldots] \\
t & =[0,1,1, n, 1,1,1,1, \ldots] \\
t & =[0,1,2, n, 1,1,1,1, \ldots] \\
t & =[0,1,1,1, n, 1,1,1,1, \ldots]
\end{aligned}
$$

Note that this selection will not resolve higher order islands lying between these two low order rationals, the largest of which is the $(3 / 5)$ island. Surfaces were deemed to be destroyed if either 
the residues of the convergents did not become small or the Fourier representation of the surface, where $M=50$ Fourier modes were used, did not lie between successive convergents. On the left of this figure the coordinate grid is shown. On both sides, Poincaré plots show the field lines that have been started on the invariant surfaces and near the unstable (1/2) and (2/3) periodic orbits. Also shown is a field line starting near the unstable $(3 / 5)$ periodic orbit. This orbit traces out the slightly chaotic $(3 / 5)$ separatrix and indicates the width of the $(3 / 5)$ island. The magnitude of the perturbation parameter $k=0.75 \times 10^{-3}$. A similar display of the coordinates, for $k=1.00 \times 10^{-3}$, is displayed in Fig.(9). For this case, in addition to the $(1 / 2)$ and $(2 / 3)$ islands, the $(3 / 5)$ island is resolved and the separatrices of the (4/7) and (5/8) islands are shown. In principle, as many islands as desired may be resolved; ultimately leading to coordinates displaying the fractal nature displayed in Fig.(5).

The extension of the coordinates from the $\phi=0$ plane to the three-dimensional volume is achieved by defining the straight field line angle $\theta_{0}$ along each of the field lines selected in Eqn.(27) as

$\theta_{0}=\alpha+t \phi$

Each surface may then be represented as a function of the $\left(\theta_{0}, \phi\right)$ coordinates, and interpolation between the surfaces enables a coordinate transformation

$\rho=\rho\left(\psi, \theta_{0}, \phi\right)$

$\theta=\theta\left(\psi, \theta_{0}, \phi\right)$

where $\psi$ is a convenient flux surface label (perhaps the toroidal flux enclosed) and the magnetic field may be written

$\mathbf{B}=B^{\psi} \mathbf{e}_{\psi}+B^{\theta_{0}} \mathbf{e}_{\theta_{0}}+B^{\phi} \mathbf{e}_{\phi}$. 
On the invariant surfaces $B^{\psi}=0$ and by using $\nabla \cdot \mathbf{B}=0$, the magnetic field may be written in the straight-field-line form

$\mathbf{B}=\nabla \psi \times \nabla \theta_{0}+t_{t}(\psi) \nabla \phi \times \nabla \psi$

Finally, if an alternative toroidal angle is desired (to simplify the representation of the magnetic field or impose a desired Jacobian) $\phi \rightarrow \phi+\delta \phi\left(\psi, \theta_{0}, \phi\right)$, then the straight-field-line poloidal angle becomes $\theta_{0} \rightarrow \theta_{0}+t \delta \phi$.

\section{Comments}

In many applications, the location of a selected irrational surface may be estimated from the location of the convergents. This estimate may be sufficient for field line following techniques to determine the magnetic surface or interest. Even if the surface located does not have exactly the selected rotational-transform, a small error may be tolerable as this region of space is likely to be filled with flux surfaces.

Incorporated into an iterative procedure [12], this construction of magnetic coordinates presented has the advantage that the 'same' surface, as defined by the value of the rotational-transform, will be constructed at each iteration. This may have benefits for numerical stability, particularly near where singularities (in the parallel current) exist. Also, a good initial guess for the trial curve may be provided by the previous iteration.

If the $\left[a_{0}, a_{1}+1\right],\left[a_{0}, a_{1}\right]$ islands are large, then it is likely a significant $\left[a_{0}, a_{1}, 2\right]$ island will form. It is possible to overlook this island. Techniques for determining island width, such as the method described in Ref.[28], may be employed to determine if an overlooked island has significant width. 
The selection of surfaces assumes some knowledge of the rotational-transform profile, perhaps from the nearby integrable field (or from a previous iteration). If insufficient information about the profile is known, some field line tracing may be necessary to obtain an estimate profile. If the rotational-transform profile is not monotonic, the mapping becomes a non-twist map.

If the field is not stellarator symmetric, all of the above ideas are applicable with the following modifications: (i) the search for periodic orbits becomes a two dimensional search and field lines must be followed the full periodic distance; and (ii) the Fourier representation of the trial curve must include all the sine and cosine terms. These modifications add to the computational effort, but do not represent any fundamental limitation of the procedure.

The author thanks Vanessa Robins, Allan Boozer, Jim Hanson, Don Monticello, Neil Pomphrey and Bob Dewar for constructive discussions. This work was supported in part by U.S. Department of Energy Contract No. DE-AC02-76CH03073. 


\section{References}

[1] J.R. Cary and R.G. Littlejohn. Annals of Phys. 151,1 (1983)

[2] W.D. D'haeseleer, W.N.G. Hitchon, J.D. Callen, and J.L. Shohet. Flux Coordinates and Magnetic Field Structure. Springer, Berlin, 1991.

[3] A.J. Lichtenberg and M.A. Lieberman. Regular and Chaotic Dynamics, 2nd ed. SpringerVerlag, New York, 1992.

[4] D.K. Arrowsmith and C.M. Place. An introduction to Dynamical Systems. Cambridge University Press, Cambridge, U.K., 1991.

[5] H. Goldstein. Classical Mechanics 2nd ed. Addison-Wesley, Massachusetts, 1980.

[6] A.H. Boozer. Phys. Fluids 24,1999 (1981)

[7] A.N. Kolmogorov. Dokl. Akad. Nauk. SSR 98,469 (1954)

[8] V.I. Arnold. Russ. Math. Surv. 18,9 (1963)

[9] J. Moser. Nachr. Akad. Wiss. Göttingen, Math. Phys. Kl. II 1,1 Kl,1 (1962)

[10] R.G Bartle and D.R. Sherbet. Introduction to real analysis. John Wiley and Sons, 1982.

[11] I. Niven. Irrational Numbers. The mathematical association of America, 1956.

[12] A.H. Reiman and H.S. Greenside. Comp. Phys. Comm. 43,157 (1986)

[13] K. Harafuji, T. Hayashi, and T. Sato. J.Comp. Phys. 81,169 (1989)

[14] L.E. Sugiyama, W. Park, H.R. Strauss, S.R. Hudson, D. Stutman, and X-Z. Tang. Nucl. Fus. $41,739(2001)$ 
[15] C.R. Sovinec, T.A. Gianakon, E.D. Held, S.E. Kruger, and D.D. Schnack. Phys. Plasmas $10,1727(2003)$

[16] J.D. Meiss. Physica D 74,254 (1994)

[17] A.H. Reiman and H.S. Greenside. J.Comp. Phys. 75,423 (1988)

[18] J. Laskar. Physica D 67,253 (1992)

[19] J. Laskar, C. Froeschlé, and A. Celleti. Physica D 56,253 (1992)

[20] R.L. Dewar and S.R. Hudson. Physica D 112,275 (1998)

[21] J.M. Greene. J.Math. Phys. 20,1183 (1979)

[22] del Castillo, D. Negrete, J.M. Greene, and P.J. Morrison. Physica D 91,1 (1996)

[23] J.D. Meiss. Rev. Modern Physics, 64,795 (1992)

[24] R.S. MacKay. Nonlinearity 5,161 (1992)

[25] B. Chirikov. Physics Reports 52,263 (1979)

[26] P.J. Morrison. Phys. Plasmas 7,2279 (2000)

[27] J.D. Hanson and J.R. Cary. Phys. Fluids 27,767 (1984)

[28] J.R. Cary and J.D. Hanson. Phys. Fluids B 3,1006 (1991)

[29] J.M. Greene, R.S. MacKay, and J. Stark. Physica D 21,267 (1986)

[30] R.S. MacKay and J. Stark. Nonlinearity 5,867 (1992)

[31] S. Marmi and J. Stark. Nonlinearity 5,743 (1992) 
[32] R.L. Dewar and J.D. Meiss. Physica D 57,476 (1992)

[33] A.H. Reiman and N. Pomphrey. J.Comp. Phys. 94,225 (1991)

[34] W.H. Press, B.P. Flannery, S.A. Teukolsky, and W.T. Vetterling. Numerical Recipes in Fortran 77: The art of scientific computing. Cambridge University Press, Cambridge, U.K., 2nd edition, 1992. 
Table I: Irrational Sequences : the irrationals given by the continued fraction representation converge to the limiting rational, from above or below, as $n \rightarrow \infty$.

\begin{tabular}{ccl}
\hline \hline & limit & continued fraction \\
\hline 1. & $(1 / 2)^{+}$ & {$[0,1,1, n, 1,1,1,1,1, \ldots]$} \\
2. & $(5 / 9)^{-}$ & {$[0,1,1,4, n, 1,1,1,1, \ldots]$} \\
3. & $(5 / 9)^{+}$ & {$[0,1,1,3,1, n, 1,1,1, \ldots]$} \\
4. & $(4 / 7)^{-}$ & {$[0,1,1,3, n, 1,1,1,1, \ldots]$} \\
5. & $(4 / 7)^{+}$ & {$[0,1,1,2,1, n, 1,1,1, \ldots]$} \\
6. & $(7 / 12)^{-}$ & {$[0,1,1,2,1,1, n, 1,1, \ldots]$} \\
7. & $(7 / 12)^{+}$ & {$[0,1,1,2,2, n, 1,1,1, \ldots]$} \\
8. & $(3 / 5)^{-}$ & {$[0,1,1,2, n, 1,1,1,1, \ldots]$} \\
9. & $(3 / 5)^{+}$ & {$[0,1,1,1,1, n, 1,1,1, \ldots]$} \\
10. & $(8 / 13)^{-}$ & {$[0,1,1,1,1,2, n, 1,1, \ldots]$} \\
11. & $(8 / 13)^{+}$ & {$[0,1,1,1,1,1,1, n, 1, \ldots]$} \\
12. & $(5 / 8)^{-}$ & {$[0,1,1,1,1,1, n, 1,1, \ldots]$} \\
13. & $(5 / 8)^{+}$ & {$[0,1,1,1,2, n, 1,1,1, \ldots]$} \\
14. & $(7 / 11)^{-}$ & {$[0,1,1,1,2,1, n, 1,1, \ldots]$} \\
15. & $(7 / 11)^{+}$ & {$[0,1,1,1,3, n, 1,1,1, \ldots]$} \\
16. & $(2 / 3)^{-}$ & {$[0,1,1,1, n, 1,1,1,1, \ldots]$} \\
\hline \hline
\end{tabular}




\section{CAPTIONS}

1. Residue below $(k=0.0015701 \ldots$, solid $)$, near $(k=0.0015801 \ldots$, dotted $)$, and above $(k=$ $0.0015901 \ldots$, dashed) criticality for the $\gamma^{-1}$ convergents.

2. Poincaré plot showing the $\gamma^{-1}$ surface, and the periodic orbits corresponding to its convergents, below criticality $(k=0.0015701 \ldots)$. The horizontal (angle) scale is $[3.117,3.166]$ and the vertical (radial) scale is $[0.63165,0.63185]$.

3. Poincaré plot showing the $\gamma^{-1}$ surface, and the periodic orbits corresponding to its convergents, near criticality $(k=0.0015801 \ldots)$. The horizontal (angle) scale is $[3.117,3.166]$ and the vertical (radial) scale is $[0.63165,0.63185]$.

4. Poincaré plot showing the $\gamma^{-1}$ 'surface', and the periodic orbits corresponding to its convergents, after destruction $(k=0.0015901 \ldots)$ : horizontal (angle) range $=[3.117,3.166]$, vertical (radial) range $=[0.63165,0.63185]$.

5. Critical perturbation parameter, $k$, for destruction of invariant surfaces, with irrational rotational-transform given in Table (I), plotted against the rotational-transform. The vertical scale is $10^{-3}$.

6. Critical perturbation parameter, $k$, for destruction of invariant surfaces, with irrational rotational-transform given in Eqn.(24), plotted against the rotational-transform. Irrationals defined by the sequence with $j=1$ are shown with ' + ', for $j=5$ with ' $\times$ ', and for $j=10$ with ' - '. The vertical scale is $10^{-3}$.

7. Magnetic coordinates (left), boundary surface and Poincaré plot near unstable $(2,3)$ periodic orbit : horizontal (angle) range $=[0.963,1.131]$, vertical (radial) range $=[0.662,0.665]$. 
8. Magnetic coordinates (left) and Poincaré plot : horizontal (angle) range $=[0,2 \pi]$, vertical (radial) range $=[0.440,0.715]$.

9. Magnetic coordinates and Poincaré plot : horizontal (angle) range $=[0,2 \pi]$, vertical (radial) range $=[0.440,0.715]$ 


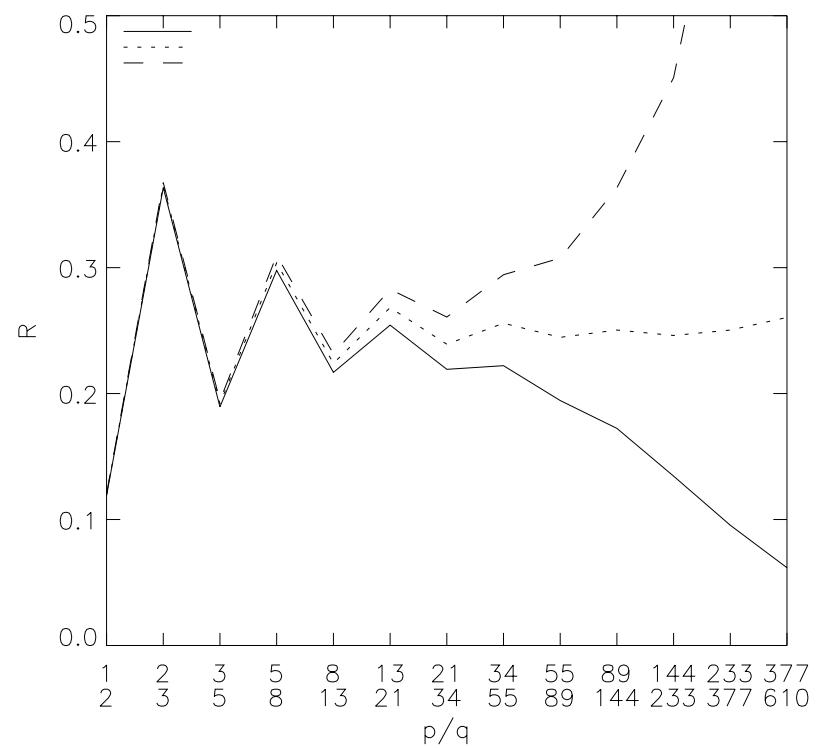

Figure 1: 


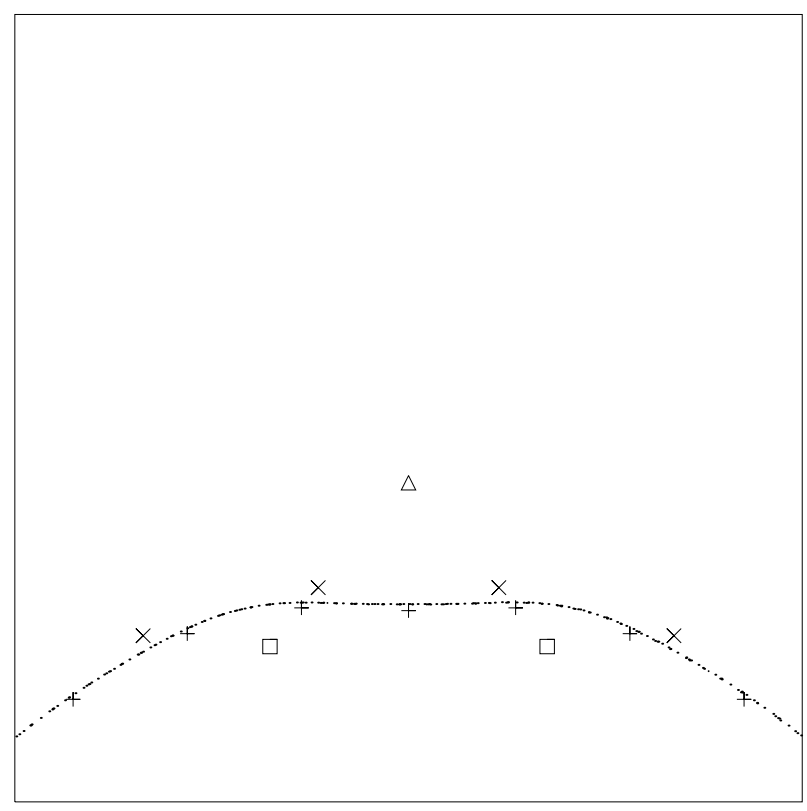

Figure 2: 


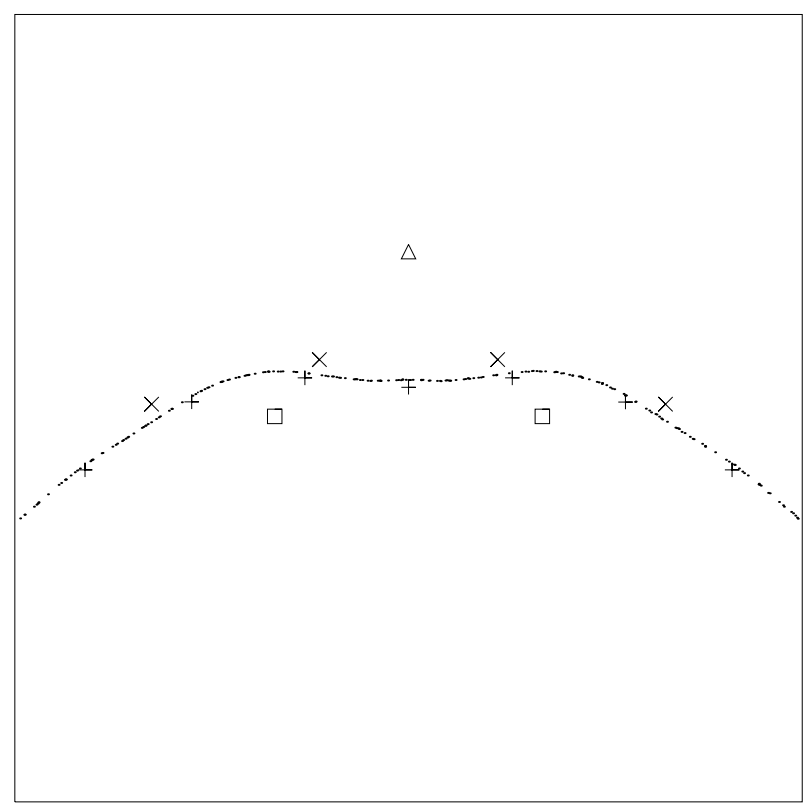

Figure 3: 


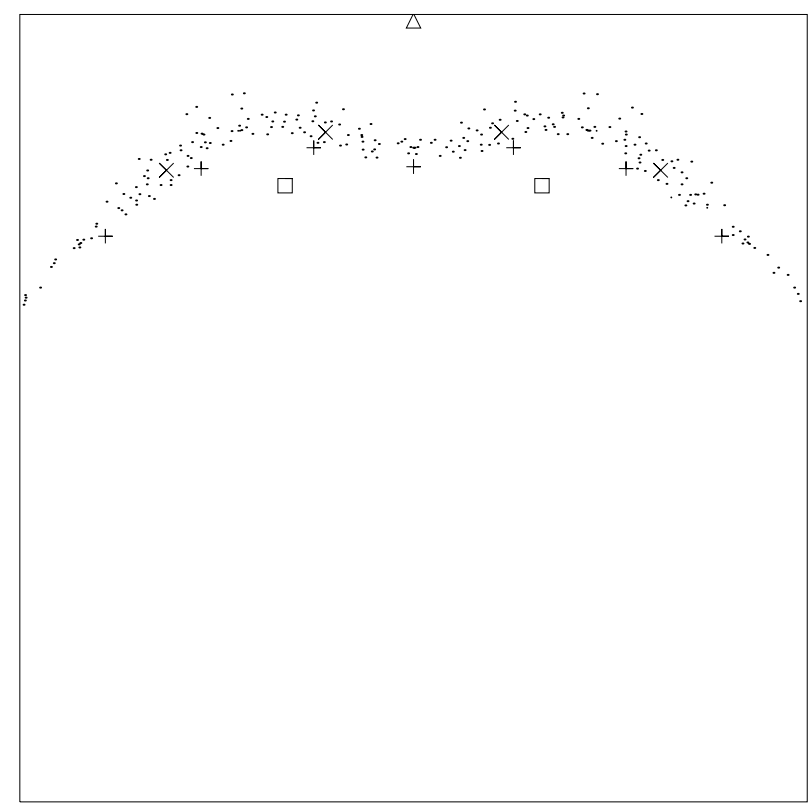

Figure 4: 


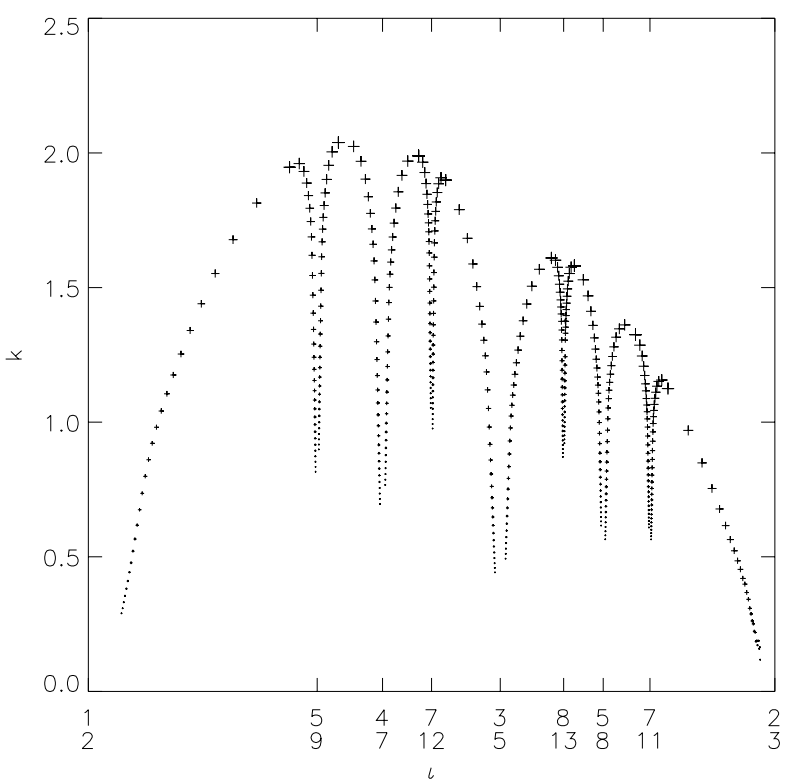

Figure 5: 


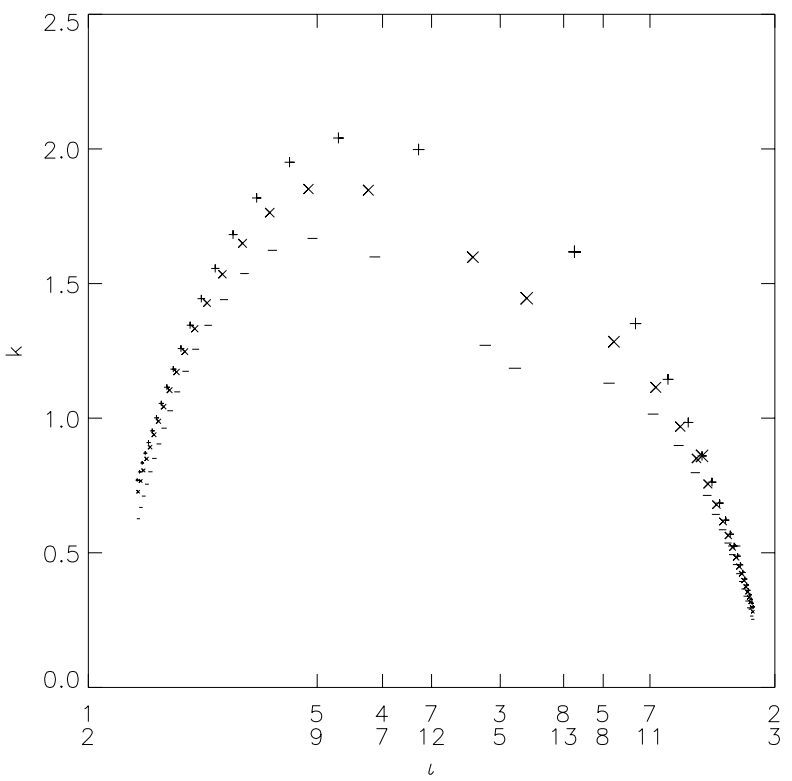

Figure 6: 


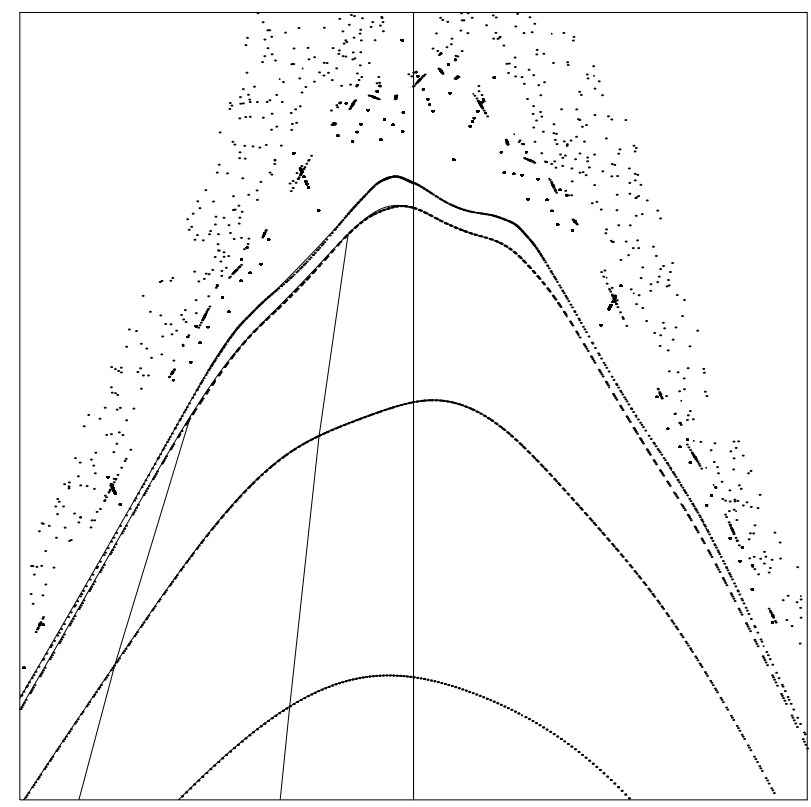

Figure 7: 


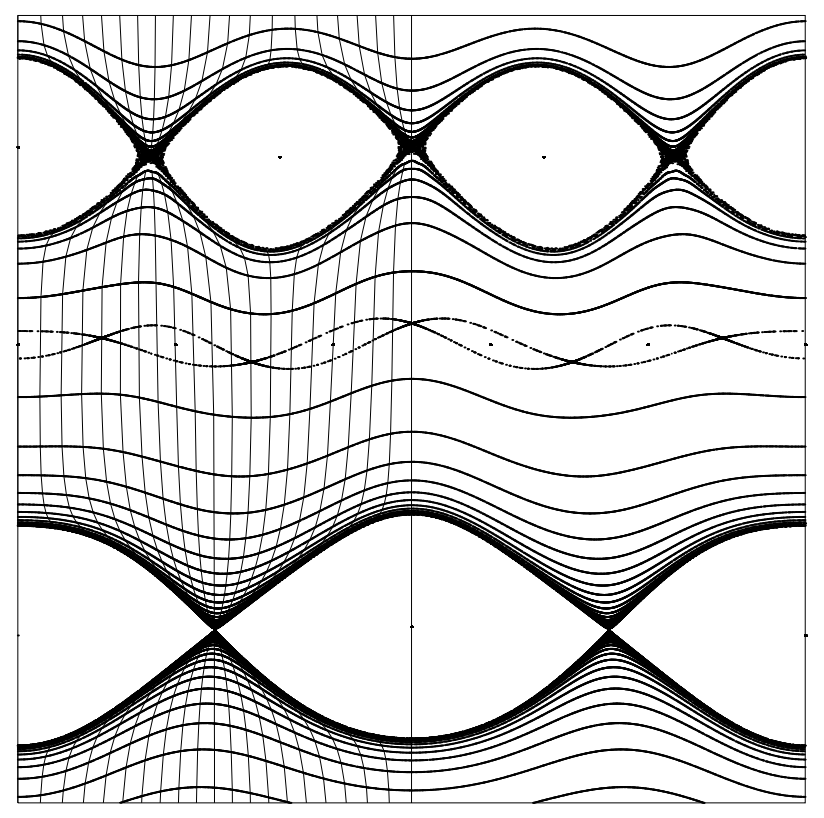

Figure 8: 


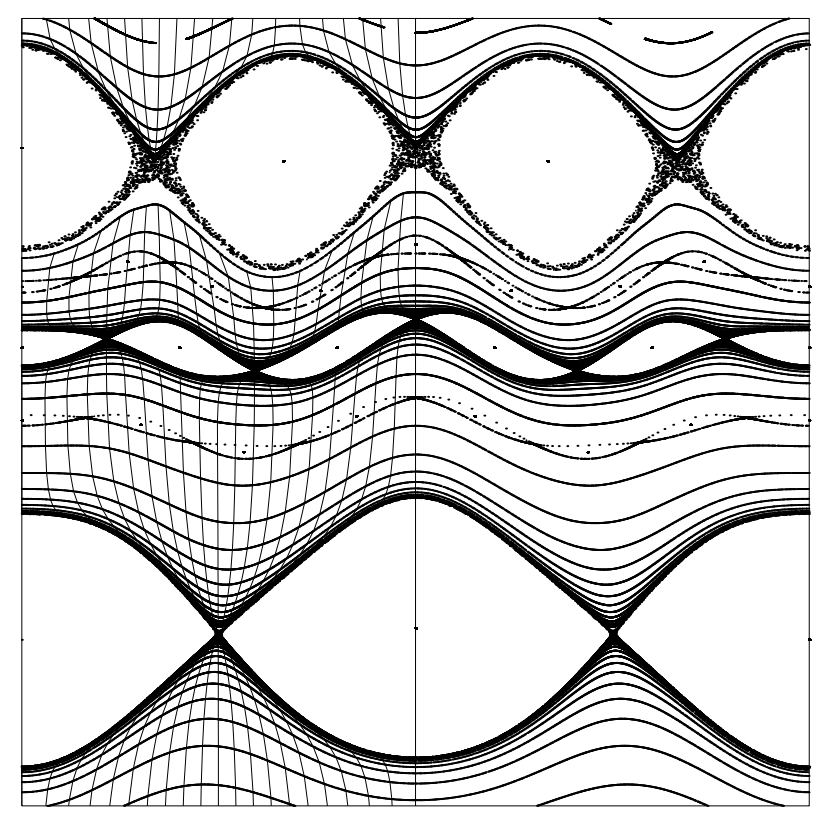

Figure 9: 


\section{External Distribution}

Plasma Research Laboratory, Australian National University, Australia

Professor I.R. Jones, Flinders University, Australia

Professor João Canalle, Instituto de Fisica DEQ/IF - UERJ, Brazil

Mr. Gerson O. Ludwig, Instituto Nacional de Pesquisas, Brazil

Dr. P.H. Sakanaka, Instituto Fisica, Brazil

The Librarian, Culham Laboratory, England

Mrs. S.A. Hutchinson, JET Library, England

Professor M.N. Bussac, Ecole Polytechnique, France

Librarian, Max-Planck-Institut für Plasmaphysik, Germany

Jolan Moldvai, Reports Library, Hungarian Academy of Sciences, Central Research Institute for Physics, Hungary

Dr. P. Kaw, Institute for Plasma Research, India

Ms. P.J. Pathak, Librarian, Institute for Plasma Research, India

Ms. Clelia De Palo, Associazione EURATOM-ENEA, Italy

Dr. G. Grosso, Instituto di Fisica del Plasma, Italy

Librarian, Naka Fusion Research Establishment, JAERI, Japan

Library, Laboratory for Complex Energy Processes, Institute for Advanced Study, Kyoto University, Japan

Research Information Center, National Institute for Fusion Science, Japan

Dr. O. Mitarai, Kyushu Tokai University, Japan

Dr. Jiangang Li, Institute of Plasma Physics, Chinese Academy of Sciences, People's Republic of China

Professor Yuping Huo, School of Physical Science and Technology, People's Republic of China

Library, Academia Sinica, Institute of Plasma Physics, People's Republic of China

Librarian, Institute of Physics, Chinese Academy of Sciences, People's Republic of China

Dr. S. Mirnov, TRINITI, Troitsk, Russian Federation, Russia

Dr. V.S. Strelkov, Kurchatov Institute, Russian Federation, Russia

Professor Peter Lukac, Katedra Fyziky Plazmy MFF UK, Mlynska dolina F-2, Komenskeho Univerzita, SK-842 15 Bratislava, Slovakia

Dr. G.S. Lee, Korea Basic Science Institute, South Korea

Institute for Plasma Research, University of Maryland, USA

Librarian, Fusion Energy Division, Oak Ridge National Laboratory, USA

Librarian, Institute of Fusion Studies, University of Texas, USA

Librarian, Magnetic Fusion Program, Lawrence Livermore National Laboratory, USA

Library, General Atomics, USA

Plasma Physics Group, Fusion Energy Research Program, University of California at San Diego, USA

Plasma Physics Library, Columbia University, USA

Alkesh Punjabi, Center for Fusion Research and Training, Hampton University, USA

Dr. W.M. Stacey, Fusion Research Center, Georgia Institute of Technology, USA

Dr. John Willis, U.S. Department of Energy, Office of Fusion Energy Sciences, USA

Mr. Paul H. Wright, Indianapolis, Indiana, USA 
The Princeton Plasma Physics Laboratory is operated by Princeton University under contract with the U.S. Department of Energy.

\author{
Information Services \\ Princeton Plasma Physics Laboratory \\ P.O. Box 451 \\ Princeton, NJ 08543
}

Phone: 609-243-2750

Fax: 609-243-2751

e-mail: pppl_info@pppl.gov

Internet Address: http://www.pppl.gov 